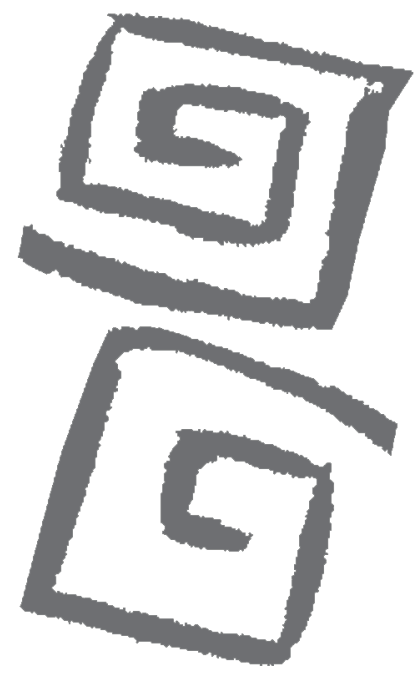

\title{
"Nosotros somos de harina": Prácticas y significados alimentarios en la Villa 21-24 de la Ciudad Autónoma de Buenos Aires, en contextos de COVID-19
}

\author{
"We are made of flour": Food practices and meanings \\ in Villa 21-24, City of Buenos Aires, in the context of \\ COVID-19
}

Gloria Sammartino ${ }^{1}$, Sonia Ana Naumann²

${ }^{\top}$ Doctora en Antropología. Becaria Posdoctoral, Consejo Nacional de Investigaciones Científicas y Técnicas. Directora, Centro de Investigación sobre Problemáticas Alimentarias y Nutricionales (CISPAN), Facultad de Medicina, Universidad de Buenos Aires. Profesora, Facultad de Medicina, Universidad de Buenos Aires. Ciudad Autónoma de Buenos Aires, Argentina. $\triangle$ iD

${ }^{2}$ Magíster en Salud Pública. Codirectora, Centro de Investigación sobre Problemáticas Alimentarias y Nutricionales (CISPAN), Facultad de Medicina, Universidad de Buenos Aires. Profesora Asociada Autorizada, Facultad de Medicina, Universidad de Buenos Aires. Ciudad Autónoma de Buenos Aires, Argentina. $\triangle$ iD
RESUMEN Se analizan los aspectos que inciden en el acceso y consumo de alimentos, con especial énfasis en aquellos obesogénicos, por parte de grupos domésticos, durante el aislamiento social a causa del COVID-19, en la Villa 21-24 de la Ciudad de Buenos Aires, en los meses de abril y junio de 2020. En un contexto atravesado por graves índices de malnutrición por exceso y por pobreza emerge el peso que poseen los entornos en los que abundan los productos industrializados, sobre todo harinas, y ultraprocesados a través de los distintos canales de acceso. La relación entre el rol de la mujer y la gestión alimentaria intrahogar muestra que quien asume la carga del hambre son las mujeres. La disponibilidad alimentaria está asegurada sin cuestionar su calidad, pero el acceso a los alimentos depende de la gestión individual, invisibilizándose como sujetos de derechos, con especial impacto en niñas, niños y adolescentes.

PALABRAS CLAVES Malnutrición; Desigualdad Social; COVID-19; Derecho a la Alimentación; Género; Argentina.

\footnotetext{
ABSTRACT This article analyzes factors affecting food access and consumption on the part of domestic groups during COVID-19 lockdown in Villa 21-24 of the City of Buenos Aires during April and June 2020. In a context marked by high rates of poverty and malnutrition due to excess, it was possible to observe the influence of food environments with abundant ultra-processed and industrialized products, especially flour, through different access channels. The relationship between the role of women and intra-household food management shows that those who bear the burden of hunger are women. Food availability is assured without questioning its quality, but access to food depends on individual management, rendering them invisible as rights-bearing subjects, with a particular impact on children and adolescents.
}

KEY WORDS Malnutrition; Social Inequality; COVID-19; Right to Food; Gender; Argentina. 


\section{INTRODUCCIÓN}

La superposición del hambre, la inseguridad alimentaria, el sobrepeso y la obesidad son los principales problemas de salud pública, y vulneran el derecho humano a la salud y a la alimentación ${ }^{(1)}$. Expresa problemas de malnutrición ${ }^{(2)}$ asociados al cambio climático como la "sindemia global", una sinergia de epidemias que comparten causas comunes fruto de los actuales sistemas alimentarios poco saludables y mínimamente regulados ${ }^{(3,4)}$ que se expresan en los entornos obesogénicos ${ }^{(5,6,7,8)}$, vulnerando la protección del derecho a una alimentación adecuada ${ }^{(9,10)}$. Es el escenario en el que cobran forma la sindemia y la pandemia del COVID-19, agudizando situaciones de desigualdad e inequidad ${ }^{(11)}$. El Informe Especial COVID-19 No. 3 de la CEPAL advierte el aumento de la pobreza por ingresos y vulnerabilidad en personas que tienen empleos informales bajo condiciones de escasez, residen en zonas periféricas, presentan alguna discapacidad o son migrantes, habitantes de calle, micro y pequeños empresarios y mujeres en situación de empleo precario ${ }^{(12)}$.

El estudio que aquí se presenta se llevó adelante en el momento en que la pobreza alcanzaba en Argentina al 40,9\% de las personas, de las cuales el 10,5\% estaba en la indigencia y entre los menores de 15 años, el $56,3 \%$ se encontraba en la pobreza total, y el $15,6 \%$ en la indigencia ${ }^{(13)}$. Estos datos empeoraron hacia el segundo semestre de 2020, con el $57,7 \%$ de las personas de 0 a 14 años estaban en condiciones de pobreza ${ }^{(14)}$. La variación interanual de la valorización mensual de la canasta básica alimentaria y de la canasta básica total para un hogar de cuatro integrantes en el Gran Buenos Aires, entre mayo 2019 y 2020 , se había incrementado el $47,9 \%$ y el $42 \%$ respectivamente ${ }^{(15)}$. Durante el estudio, la pronunciada suba de precios en los alimentos y demás rubros básicos se vio agravada por la situación epidemiológica y sociosanitaria para la población en general y, en especial, para la población infantil ${ }^{(16)}$.

La situación alimentaria y nutricional poblacional ya era compleja, la prevalencia de sobrepeso si bien afecta a todos los grupos de edad y los quintiles de ingresos, los grupos sociales de menores ingresos evidenciaron mayores índices de exceso de peso, con un $21 \%$ mayor en el quintil de ingresos más bajos respecto del más alto ${ }^{(17)}$, lo cual se recrudece ante el impacto del COVID-19, vulnerando aún más los derechos humanos ${ }^{(18,19,20)}$. El estudio fue realizado desde un enfoque cualitativo que asume los procesos alimentarios desde una perspectiva holista ${ }^{(21,22)}$, atravesados por relaciones de poder que inciden en los significados que poseen los alimentos para las personas ${ }^{(23)}$. Se prestó atención a las relaciones entre la malnutrición por exceso y el lugar de las mujeres. Cabe señalar que, si bien en Argentina la mayor prevalencia de sobrepeso se registra en los varones $(37,5 \%)$ con respecto a las mujeres $(31,1 \%)^{(24)}$, la tendencia en el aumento de la obesidad ha impactado de manera desproporcionada en las mujeres de América Latina y el Caribe: en más de 20 países de la región la tasa de obesidad femenina es casi diez puntos porcentuales mayor que la de los hombres ${ }^{(25)}$. Asimismo, la prevalencia de inseguridad alimentaria es mayor en mujeres que en hombres, lo que ubica a Argentina entre los países que mantienen las mayores brechas entre hombres y mujeres $^{(19)}$. Estas estadísticas refuerzan la importancia de incluir la perspectiva de género, la cual permite visibilizar que las mujeres asumen la mayor parte de la carga del trabajo social reproductivo, incluidas las "responsabilidades" concernientes a la alimentación, que reproducen estructuras de desigualdad y de poder ${ }^{(26,27)}$. Se encuentran entre los grupos más vulnerables respecto a la discriminación de género; a las restricciones sociales, legales y culturales; disponen de un acceso más limitado que los hombres a las oportunidades de trabajo, los recursos productivos y financieros, la educación, la toma de decisiones y los mercados laborales ${ }^{(27)}$. En este marco la interseccionalidad es un enfoque clave para describir cómo grupos de personas se encuentran en desventaja debido a múltiples fuentes de prejuicios y discriminación, por sus identidades y experiencias superpuestas, como raza, clase, identidad de género, etc. ${ }^{(28)}$. 
El concepto de raza alude a la existencia de grupos históricamente excluidos en el marco de una estructura y matriz colonial de poder racializado y jerarquizado, con los blancos y "blanqueados" en la cima y los demás pueblos (indígenas, campesinos, afrodescendientes, etc.) en los peldaños inferiores ${ }^{(29)}$. Esta estructura se conecta con los procesos de estigmatización sobre "los pobres" y "su cultura"(30), que refuerzan prejuicios preexistentes hacia quienes pertenecen a sectores más desfavorecidos $^{(31)}$, y se expresan en discursos racistas y xenófobos, entre los que se incluye a los residentes de países limítrofes ${ }^{(32)}$.

Tomamos asimismo las necesidades de cuidado como algo que afecta a todos los seres humanos en todas las etapas de la vida y como parte esencial de la reproducción social, las cuales permiten visualizar cómo el cuidado se reparte entre géneros, generaciones, familia, Estado y mercado. Este planteamiento posibilita profundizar en las relaciones y tensiones entre las desigualdades entre hombres y mujeres en el campo de la alimentación y la malnutrición por exceso, en particular ${ }^{(24)}$.

El artículo describe aspectos y significados que inciden en el acceso y el consumo de alimentos, con énfasis en productos ultraprocesados, de grupos domésticos en contexto de pandemia por COVID-19, en la Villa 21-24 (en adelante barrio 21/24) de la Ciudad Autónoma de Buenos Aires (CABA), durante los meses de abril y junio de 2020. Entre los aspectos analizados se incluyen los canales de acceso a los alimentos y las comidas, la identificación de responsables de la alimentación al interior de los hogares, los criterios de compra de alimentos, los alimentos considerados más importantes y los posibles obstáculos que inciden en su adquisición, preparación y consumo.

\section{ASPECTOS METODOLÓGICOS}

El trabajo se desprende de un estudio mayor, observacional, descriptivo y de corte transversal, de enfoque cuali-cuantitativo, que describe aspectos de los entornos, las unidades domésticas del Área Metropolitana de Buenos Aires y la relación con el estado nutricional de sus integrantes. Dicho estudio se enmarca en el proyecto "Alimentación y entornos obesogénicos: Estudio desde una mirada multidisciplinaria en contextos urbanos y periurbanos de Buenos Aires" (UBACyT 2018-2020 y Beca Salud Investiga Dr. Abraam Sonis para estudios multicéntricos 2018). El estudio, realizado entre 2018 y 2019, incluyó población que presentaba condiciones socioeconómicas desfavorables, residente del Área Metropolitana de Buenos Aires, entre ellas, el barrio 21/24, ubicado en la Comuna 4 de la Ciudad Autónoma de Buenos Aires. Entre algunas de sus características, se destacan altos niveles de hacinamiento, falta de red de gas, cloaca y agua potable en muchos hogares y, en el caso del gas, la población lo utilizaba mayormente envasado. La mayoría de las personas adultas formaba parte del mercado de trabajo no registrado ${ }^{(33,34,35)}$. Otra característica fue la marcada presencia de inmigrantes provenientes de Paraguay. Si bien no hay datos oficiales, se pudo observar la presencia de múltiples instituciones, de puestos de comidas y productos regionales.

Se trabajó con hogares compuestos por personas adultas $y$ al menos un niño, niña y/o adolescentes de entre 5 y 19 años que asistieran a uno de los merenderos autogestionados, con cuyo referente teníamos contactos previos. Se incluyeron unidades domésticas en las que respondiera la persona responsable de las compras de alimentos y conviviera con al menos un niño, niña y/o adolescentes de entre 5 y 19 años. Se excluyeron del estudio las personas adultas que presentaran alguna enfermedad incapacitante para responder $y / o$ completar las fases del estudio. Las unidades domésticas fueron seleccionadas por muestreo no probabilístico por conveniencia. Se realizaron entrevistas en profundidad, alternando técnicas como entrevistas informales y observación participante ${ }^{(36)}$.

El estudio se enmarca en los aspectos éticos de autonomía, beneficencia y justicia $^{(37)}$, fue evaluado y aceptado por el comité de ética del Hospital "Prof. Dr. Juan P. 
Garrahan". Todas las personas que participaron del estudio firmaron el correspondiente consentimiento informado. Los nombres originales fueron cambiados para preservar la confidencialidad de los datos.

Al inicio de la pandemia, se planteó otro estudio, realizado con el apoyo de UNICEF Argentina, de corte exclusivamente cualitativo, fenomenológico interpretativo, titulado "Prácticas alimentarias, obesidad y COVID-19 en contextos de pobreza urbana: Villa 21-24, Ciudad de Buenos Aires, Argentina, 2020". Para este estudio, se volvió a contactar al referente barrial del merendero y a 17 mujeres que integraban los grupos domésticos del barrio 21/24 con quienes se había trabajado y se mantenía contacto, todas ellas conviviendo con al menos un niño, niña y/o adolescente, y asistentes al merendero mencionado. Se llevaron a cabo entrevistas en profundidad, de una hora aproximada de duración, entre abril y junio del año 2020 a través de Ilamadas al celular personal de las mujeres. Si bien la metodología cualitativa tradicionalmente está basada en las relaciones cara a cara(38), debido al confinamiento obligatorio esta modalidad fue la posible. Las entrevistas fueron grabadas. Tras su desgrabación, transcripción y lectura, se construyeron las matrices cualitativas con el análisis de los fragmentos incluidos en las categorías para establecer los ejes conceptuales y la construcción del texto.

\section{RESULTADOS}

\section{Ambiente alimentario y acceso a los alimentos en el barrio 21/24}

Del análisis de las entrevistas resulta que las edades fueron entre 18 y 55 años. Ninguna de las personas, al momento de las entrevistas, había sido diagnosticada con COVID-19; aunque una de las mujeres, que estaba planeado entrevistar, murió diagnosticada de COVID-19. Prácticamente la totalidad de las entrevistadas estaban a cargo de las tareas domésticas y de cuidado, algunas de manera exclusiva por ser las únicas adultas responsables y otras, en distintos grados, compartiéndolas con sus parejas. Sus situaciones económicas presentaban distintos grados de vulnerabilidad. El desempleo, la suspensión de actividades laborales de tipo informal, la suba de precios, el miedo al contagio, la baja calidad de los servicios de Internet, (que constituía un obstáculo para que niños, niñas y adolescentes tomaran clases virtuales) se fueron agudizando conforme avanzaba el confinamiento. Sobre los canales de acceso a los alimentos y comidas se hallaron tres:

1) Comercios: hipermercados, supermercados y almacenes, verdulerías, carnicerías y panaderías del barrio.

2) Asistencia alimentaria del Estado: programas de transferencia directa de dinero como Asignación Universal por Hijo (AUH) ${ }^{(39)}$, Ingreso Familiar de Emergencia de la Administración Nacional de la Seguridad Social de $\$ 10.000$ (IFE) $^{(40)}$ y Tarjeta Ciudadanía Porteña ${ }^{(41)}$; comedores comunitarios que recibían alimentos para preparar viandas para retirar, y escuelas públicas en las que quincenalmente y por alumno/a se retiraban módulos de alimentos provenientes del Programa de Educación Escolar ${ }^{(42)}$. Respecto a la Tarjeta Alimentar, su falta de mención responde al hecho de que, en la Ciudad Autónoma de Buenos Aires, al momento de realizar el trabajo de campo, la gente no la estaba recibiendo.

3) Organizaciones sociales, de tipo barriales, iglesia, universidades, vecinos autoconvocados, etc., que canalizaban acciones de recepción, preparación y distribución de alimentos donados. Sobre el contenido del módulo escolar, se registró: leche entera larga vida, fideos, arroz, lentejas, arvejas u otras legumbres, aceite, puré de tomate, pescado en conserva, queso rallado, flan o postre de leche, azúcar, zanahoria, ceboIlas, zapallo y frutas. Queda en evidencia la combinación por parte de las familias de los distintos canales de acceso tal como surge en testimonios: 
...yo recibo una bolsa por cada uno [de los cuatro hijos escolarizados], o sea cuatro bolsas, cuatro leches y después lo que es verduras, papa, cebolla, zanahoria, menos morrón, zapallo, calabaza, y con eso nos mantenemos, más la mercadería que yo compro. Lo único que me queda es la carne o el puré de tomate que es lo que más uso. (Inés, 38 años, madre de 5 hijos)

El principal criterio de compra en este contexto sociosanitario referido por las entrevistadas es la optimización del recurso económico disponible, combinado con la búsqueda de alimentos considerados básicos y que "no podrían faltar". El principal alimento es la carne (fundamentalmente pollo -pata y muslo y alitas-, también vacuna -carnaza, osobuco, carne picada, mondongo-), de compra quincenal y mensual, en algunos hogares almacenados en freezers -no todos los hogares disponían- de manera fraccionada y adquirida en super e hipermercados. Dicha modalidad fue la más mencionada, pero no todos los grupos domésticos pudieron efectivizarse por falta de recursos. También pan, harina, algunas verduras y frutas, leche y yogurt bebible, galletitas dulces, productos de snack, golosinas y bebidas azucaradas. Algunas entrevistadas refirieron resolver compras de urgencia en los comercios barriales, destacando la practicidad basada en la proximidad a expensas de pagar precios altos comparados con los de las cadenas de hipermercados y de supermercados que se encuentran entre uno y dos kilómetros de distancia del barrio. Los principales alimentos referidos fueron pan, algunas verduras o frutas que no hubieran recibido a través de los módulos en las escuelas o comedores, bebidas azucaradas, productos de snacks y golosinas.

Hay que ir a buscar algo de ventaja, muchísima diferencia en los precios [compara con carnicerías en el barrio] en Coto, [una conocida cadena de hipermercados] por ejemplo, sale carísimo, y no ponen lo barato como espinazo, ponen lo que un rico puede comprar, pero ahora hace años que cambiamos por Día [otra conocida cadena de supermercados], siempre buscamos ventaja y ahora más con esta situación, tenemos que ir hasta allá. (Teresa, 45 años, 3 hijos)

En los siguientes testimonios, se refleja la barrera económica como principal obstáculo de acceso a los alimentos considerados básicos por parte de algunas entrevistadas, empleadas domésticas, principal sostén del hogar, que al no poder concurrir a sus trabajos fuera del barrio, dejaron de percibir parte fundamental de sus ingresos:

Carne ya casi ya no compro, compro más pollo, alitas, trozados, carne muy poco se come ya, carne se come cuando traemos del comedor o alguna milanesa que hay, antes sí hacía mucho milanesa. (Marta, 39 años, dos hijos)

Cuesta más conseguir, yo soy siempre de tener el pan de todos los días, no hay verduras, las verduras están por las nubes, que es lo que más se usa para el guiso, y es lo más básico, zanahoria, morrón y cebolla. (Nélida, 50 años, 3 hijos)

Se observa tanto en el testimonio anterior, como en el que sigue, el lugar periférico que tienen gustos y preferencias de los menores de la familia, como la preocupación por productos de limpieza relacionada con las medidas de higiene debido a la pandemia:

Harina, aceite, cuando puedo compro pollo, si no compro lavandina. A veces le suelo comprar a los chicos lo que quieren ellos, banana, a veces. Yogurt es lo que les gusta a ellos, en Día está más o menos el precio..., Pitusas [galletitas dulces], y el surtido ese la grande es lo que le llevo más para que a ellos les alcance (María, 33 años, 4 hijos)

Las personas responsables de comprar los alimentos y retirar los bolsones y/o viandas fueron, sin excepción, las mujeres a cargo de los grupos domésticos, mientras que el lugar 
de sus compañeros es el de "ayudarlas". Respecto del rol de las organizaciones sociales, es de destacar la respuesta que otorgaron a quienes atravesaron una mayor situación de vulnerabilidad. El surgimiento de las ollas populares, merenderos y comedores, así como el marcado incremento de personas acudiendo a estos canales sobre el final de nuestro trabajo de campo, resultó coincidente con la suba de casos positivos por COVID-19 y la extensión de la cuarentena. Entre los alimentos menos valorados aparecen las legumbres y las calabazas, aduciendo la gran cantidad recibida a través de la asistencia alimentaria y la ausencia de preferencia acorde a sus hábitos alimentarios y habilidades culinarias, lo que motiva a donar, intercambiar y/o vender estos alimentos. Al respecto una de las entrevistadas comentaba que intercambiaba con su suegra garbanzos provenientes de los módulos alimentarios escolares, por harina, azúcar o leche, aduciendo que ella no sabía cocinarlos y que a sus hijas e hijos tampoco les gustaba.

\section{Nosotros somos de harina}

Respecto a las preparaciones y consumos alimentarios durante el período de confinamiento, emergen las elaboraciones habituales, previas a la pandemia, con una mayor proporción de harinas refinadas y productos con elevados aportes de azúcar, sal y grasas a expensas de alimentos naturales y frescos como frutas, verduras, carnes, quesos, huevos, entre otros. Las elaboraciones más mencionadas coincidieron con las ya hechas en el estudio anterior (2018-2019), donde se registraron guisos en sus múltiples variantes (con pollo, con carne, con arroz, con fideos, de polenta), estofados, puchero, sopas, etc. También fideos o arroz con manteca y/o queso; las milanesas, aunque asociadas en muchos casos con comidas "caras" y de excepción. En todos los casos, la asociación de dichas elaboraciones con las limitaciones económicas previas son centrales y recurrentes, tal como se plasma en el siguiente extracto de una de las entrevistas: ...no nos queda otra, economizar como uno pueda, no les gusta el guiso todos los días, pero tiene que comer si tienen hambre, yo antes llenaba la heladera, había ravioles, les hacía empanadas, de todo, ahora no, comida económica. (Nelida, 50 años, 3 hijos)

Aparejado a la cuarentena aparece más marcada la mención de elaboración de panes, tortas, y galletitas. Adquieren mención especial las tortas fritas y tortillas a las brasas, que aparecen tanto comidas a la tarde-noche, como en la última comida del día. Fueron señaladas también, por algunas mujeres, como la única opción alimentaria disponible durante semanas. A diferencia de otros sectores más privilegiados, para los que la cuarentena posiblemente pudo significar contar con tiempo y una amplia gama de ingredientes, se evidencia que las condiciones estructurales desfavorables (falta de gas, hornos, utensilios, ingredientes, etc.) incidieron notablemente en desmedro de la calidad y variedad de las preparaciones. Entre los repertorios culinarios mencionados, también registrados en el mencionado estudio anterior, aparecen comidas regionales, cuyos ingredientes también se hallaban disponibles en los comercios barriales, como bori bori, sopa paraguaya, mandioca frita, chipá guazú, chupín de pescado, elaboraciones con porotos negros, entre otros:

...bori bori, no sé si vos lo conocés, cocino la carne, le saco el jugo para mojarlo en la harina de maíz, hacés una masa, le ponés queso, y ahí hacés la bolita. (Gladys, 40 años, 1 hijo)

Hervida o podés poner a un puchero [...] Mandioca frita, hay que hervir la mandioca, cuando está blanda, hay que pasar otra vez en aceite para quedar doradita, entonces, y el huevo también hay que hacer frito. Pero eso es medio pesado. (Martina 30 años, 4 hijos)

Aunque asociadas a estas comidas aparecen alusiones a lo que da gusto comer, también 
aparece "lo pesado" y "grasoso". Sobre las preparaciones de los comedores comunitarios y de las ollas populares suelen ser similares a las mencionadas en primer lugar, sin observarse preparaciones regionales. En los merenderos redundan elaboraciones de pastelería casera y productos de panadería (facturas, pan, torta, churros), también todo tipo de galletitas y magdalenas, leche y mate cocido. Las bebidas más mencionadas que se consumen en los hogares y que se reciben en merenderos y comedores suelen ser bebidas azucaradas e industrializadas (jugos en polvo, concentrados y gaseosas). La leche también aparece como una bebida primordial, sobre todo en desayunos y meriendas, con mate cocido.

Las restricciones económicas adquieren lugar central, tal como lo señalaba una de las entrevistadas, "porque no Ilego con la plata, hice guiso de arroz, y hoy voy a hacer guiso de fideos". Esto se conecta con las posibilidades de compras de alimentos, restringidos a determinados productos frescos, prácticamente carne y una reducida variedad de hortalizas. La restricción también aparece en las cantidades de carne como estirando las comidas, el abanico de posibilidades queda reducido a distintas elaboraciones con los mismos ingredientes. A la mención recurrente de los guisos aparece en un estatus similar el consumo de harinas para hacer las tortillas y tortas fritas, los fideos, la polenta, o el reviro (que es otra de las elaboraciones de origen paraguayo cuyos ingredientes son harina y grasa).

...es harina, arroz, fideos, hago torta fritas, hago lo que nosotros les decimos reviro, nosotros lo que más consumimos es harina, es lo más barato, ahora para comprar otra comida es muy caro. (Pamela, 32 años, 4 hijos)

En este testimonio se refuerza la necesidad de que sean alimentos baratos, rendidores y saciadores ${ }^{(43)}$, lo que queda conjugado en las harinas. Por esta razón, una de las entrevistadas comentaba "nosotros somos de harina". Ante la ausencia de recursos, las preparaciones quedan restringidas a un abanico reducido de alimentos, donde las harinas y los productos que la contienen son los más fáciles de obtener y cuentan con la aceptación de todos los integrantes del hogar. Otra de las dimensiones es la del gusto, sobre todo de los niños más pequeños. Emerge una marcada preferencia por las milanesas, los fideos y el arroz "blancos", es decir, sin salsas, con manteca o aceite y/o queso. Otros de los alimentos que tienen un lugar marcado entre los gustos infantiles son las galletitas dulces para la merienda, como las frutas y el yogurt. Entre las comidas menos aceptadas se encuentran las que tienen legumbres, tal como queda reflejado en los siguientes extractos de los testimonios:
Yo lo hago, pero a los chicos no les gusta el poroto. Una vez hice con fideos, y le puse queso de campo, pero a ellos no les gusta, hago al pedo, no me comen. (Pamela, 32 años, 4 hijos)

De lo señalado emergen referencias que refuerzan el peso que tienen las harinas entre niños, niñas y adolescentes, también el gusto por las frutas y, a la vez, cierto rechazo por comidas elaboradas en los hogares con legumbres, que son las que provienen en general de los bolsones escolares.

Entre otros factores claves que inciden en la elaboración de las comidas apareció la importancia de la disponibilidad de garrafas de gas, si bien algunos hogares cuentan con hornos eléctricos, no es el caso de la mayoría. Era recurrente la mención a que cuando se acababa la garrafa y no se contaba con recursos para renovarla, era posible estar muchos días sin poder cocinar. Aunque el acceso al agua no aparece en general como un obstáculo, pues está naturalizado, en algunas viviendas el agua del grifo posee olor a cloaca. Es el caso de una de las entrevistadas, que vive en el primer piso de una edificación de varias piezas de alquiler, y que diariamente acarrea agua en bidones de la casa de una vecina, a unos doscientos metros de distancia. 


\section{A caballo regalado no se le miran los dientes}

Otra arista sobre el gusto se vincula con la calidad de las comidas del comedor, que no siempre resultan aceptables:

\begin{abstract}
...a veces se cocina mal y yo les digo, y me dicen si no te gusta no vengas, [...]. medio amargo, no es rico, a veces no hay lugar a la queja (Asunción, 49 años, dos hijos)
\end{abstract}

...hay muchos lugares que te dan así nomás la comida. Pero bueno, o siempre te dan lo mismo, te cocinan guiso todos los días y te dan guiso, guiso, guiso. (Natalia, 32 años, tres hijos)

Emerge la dificultad para expresar el descontento, pues subyace el agradecimiento tácito ante el recibimiento de comida. Se esgrimen distintas tácticas, como volver a cocinar la comida en los hogares, agregarle otros ingredientes y condimentos. Aparece la alusión recurrente respecto a niños, niñas y adolescentes que no siempre aceptan comer las comidas que sus padres traen del comedor:

\section{.... a veces los chicos no me quieren comer [...] a veces no cocinan bien y nadie quiere comer y tengo que comprar alguito para guardar en la heladera. (Pamela, 32 años, 4 hijos)}

Se realizó también una pregunta acerca de si consideraban que en el barrio la gente estaba pasando situaciones de hambre. La respuesta más generalizada fue que esto no sería posible, dada la existencia de comedores, merenderos, ollas populares y distribución de mercaderías por parte de la red de asistencia alimentaria estatal y de las mismas organizaciones sociales, tal como se señala aquí:

...acá es imposible que pases hambre. El que diga que está pasando hambre miente, porque acá es muy accesible, vas con la ollita y pedí la comida. No, no, eso de pasar hambre no. (Natalia, 32 años, 4 hijos)

...La gente acá en el barrio no se muere de hambre porque hay olla por todos lados, hay desayuno, hay almuerzo, hay merienda y hay cena, hacemos torta fritas, nos donan churros y bolitas, hay galletitas que nos donan, hay... sí, galletitas. (Liliana, 43 años, una hija)

Se desprende que las preparaciones que contienen harinas, azúcar, fideos, arroz y polenta son las que circulan en abundancia en el barrio. De la mano con esto, se remarca la invisibilización de la dimensión del gusto. También emerge que el riesgo de pasar hambre en los hogares existe ante la falta de capacidades personales para gestionar alimentos o comida, ya sea acudiendo a los distintos espacios oficiales de asistencia alimentaria, de las organizaciones sociales o canales de acceso organizados por parte de las propias personas autogestionadas que viven en el barrio. También la vergüenza, en el marco de la agudización de la falta de recursos por no percibir ingresos, debido a las medidas restrictivas vinculadas al confinamiento, de tener que salir a pedir comida:

\footnotetext{
...el problema es que mis chicos no quieren ir al comedor, no están acostumbrados, ellos dicen "si tenemos que pasar hambre pasaremos hambre todos, pero yo a un comedor no me quiero ir"... por el momento hambre no pasamos. (Asunción, 49 años, dos hijos).
}

Quedan reflejadas la incomodidad y vergüenza que enfrentan algunas familias que se empobrecieron a raíz de la crisis económica y el contexto de pandemia, lo cual resulta en una barrera a la hora de solicitar ayuda alimentaria. Otro aspecto es el lugar de responsabilidad de las mujeres, tanto para acceder a comidas fuera de sus hogares como de elaborarlas dentro de estos:

...el que pasa hambre es porque la mamá no le cocina, porque ayuda tenemos, de 
los comedores o te entregan cajas de mercadería, y la mayoria por cada chico que tiene cobran, no entiendo porque pasarían hambre. (Inés, 35 años, 4 hijos)

Esto se conecta con la alusión a la falta de habilidades culinarias por parte de las mujeres, así como la falta de interés en dichas tareas culinarias.

Se destaca la asociación referida por las mujeres entre la ausencia de clases presenciales en las escuelas y su impacto beneficioso en la situación alimentaria de sus hijos, como queda reflejado en esta frase: "ahora está comiendo bien, ahora que no va a la escuela, come". Otras señalan que sus hijos ya no comen tantas golosinas, y aparece también el alivio de no tener que sucumbir a los pedidos reiterados de estas a la salida de la escuela.

\section{DISCUSIÓN}

En esta sección pondremos en discusión los principales hallazgos organizados en cuatro áreas temáticas: el acceso a los alimentos, el consumo de alimentos, el rol de las mujeres vinculado a tareas de cuidados y alimentación familiar, y la relevancia de las organizaciones de la sociedad civil. Todas estas son áreas atravesadas por las condiciones de vida y el empobrecimiento, condiciones que se vieron maximizadas por la situación sociosanitaria causada por la pandemia ${ }^{(34)}$. A esto se suma la continuidad en la suba del precio de alimentos, el desempleo, y la imposibilidad en muchos casos de ir a trabajar con la consecuente disminución o pérdida de ingresos monetarios en los hogares ${ }^{(19,44)}$. En este contexto, el acceso a los alimentos, según las voces de las entrevistadas, revela la implicancia de los precios poco competitivos de los comercios minoristas y la competencia que ejercen sobre estos las cadenas de supermercados, las cuales poseen el control sobre la distribución de los alimentos, sobre todo de productos envasados y carne ya troceada, de manera tal de sostener ofertas y promociones ${ }^{(45)}$, que dejan en desventaja a los comercios minoristas, que no obstante logran "sacar de apuros". Otro aspecto que revela el poder de las empresas o proveedores de la esfera gubernamental, es que los módulos alimentarios se encuentran parcialmente integrados por productos ultraprocesados (como los postres lácteos) altos en nutrientes críticos. Las entrevistas dan cuenta de la incidencia positiva de las medidas del gobierno nacional, a través del pago del Ingreso Familiar de Emergencia (IFE) ${ }^{(40)}$-cuyas primeros cobros fueron simultáneos a nuestro trabajo de campo-, y las del gobierno municipal de la Ciudad Autónoma de Buenos Aires, con la entrega de los módulos alimentarios escolares. Estas medidas colaboraron a que los hogares pudieran tener un acceso mínimo de alimentos aunque, debido a la suba de su precio, la compra quedó restringida a los considerados básicos: harina, aceite, papas, cebollas y carne.

La relación entre el entorno alimentario escolar y su impacto en el acceso, comportamiento y consumo alimentario de sus hijos e hijas resultó una dimensión emergente del estudio. Si bien aparece con claridad la importancia que adquiere la asistencia alimentaria directa que la escuela brinda en general, también surge con recurrencia la noción de que "sin escuelas, los chicos comen mejor", aludiendo a que, debido al confinamiento, no se encuentran expuestos a la oferta de productos de kioskos, vendedores ambulantes y publicidades callejeras que promueven el consumo de productos alimentarios altos en nutrientes críticos en el ámbito escolar. La $2^{\circ}$ Encuesta Nacional de Nutrición y Salud (ENNyS 2) ${ }^{(17)}$ muestra que al $71,2 \%$ de niños, niñas y adolescentes escolarizados, la escuela les provee a veces o siempre facturas, productos de pastelería, galletitas dulces y/o cereales con azúcar; mientras que solo 2 de cada 10 estudiantes escolarizados reportaron que su institución siempre les provee frutas frescas. También se observó que 6 de cada 10 niños, niñas y adolescentes escolarizados de entre 6 y 17 años habían comprado golosinas en el kiosco o buffet de la escuela durante la última semana. Esto muestra la necesidad de implementar medidas de regulación de 
Ios entornos escolares, entre ellas la recientemente sancionada Ley 27642 de Promoción de la Alimentación Saludable ${ }^{(46)}$, para que niños, niñas y adolescentes cuenten con entornos alimentarios que contribuyan a garantizar el derecho a la alimentación adecuada ${ }^{(47)}$. A su vez, se observa la necesidad de regular las donaciones alimentarias, principalmente, aquellas centradas en la entrega de productos ultraprocesados, que refuerzan el patrón alimentario industrializado ya descripto.

La relación entre acceso y consumo de alimentos es estrecha. En este sentido, si bien la carne es altamente valorada ${ }^{(48)}$, al existir restricciones para su compra, queda en un lugar periférico en las elaboraciones respecto a la cantidad y calidad (abundan cortes grasos). La misma situación se da para el caso de las frutas, tienen una alta valoración, pero aparece con insistencia que su compra queda relegada. Esto encuadra con que la población perteneciente a los quintiles más bajos tiene un consumo que desciende a la mitad en relación con el más alto, $22,8 \%$ vs $45,3 \%$, respectivamente ${ }^{(17)}$. Entre otros consumos valorados por las entrevistadas como de "prestigio", aparecen el yogur, las galletitas dulces, las bebidas azucaradas artificiales, y productos de copetín (papas fritas, chizitos, palitos salados, etc.), asociados a la búsqueda de satisfacer los gustos y antojos de sus hijos e hijas, y de otros integrantes del hogar. Es de resaltar que el consumo de bebidas artificiales con azúcar y los productos de copetín en la misma ENNyS 2 muestra ser dos veces mayor en los quintiles más bajos que en los más altos.

Tanto el acceso como el consumo de los alimentos, se enmarcan en las características de los entornos obesogénicos signados por el aumento del consumo de productos ultraprocesados $^{(49,50)}$, impulsados por estrategias no reguladas de marketing que "secuestran el paladar", orientadas a la población infanti $\left.\right|^{(51)}$. Se hallan conexiones entre el aumento en la disponibilidad de productos ultraprocesados ${ }^{(7,52)}$, la configuración del sistema agroalimentario hegemónico actual(53) y la falta u omisión de políticas públicas que puedan moldear entornos alimentarios cada vez más saludables y sostenibles ${ }^{(52)}$ para la población general y para la población infantil en particular. Esto se condice con los estudios locales que muestran el aumento en la compra ${ }^{(7)}$ y consumo ${ }^{(54)}$ de estos productos en Argentina. Siendo importante el peso de la cadena agroindustrial dentro de la economía global de mercado(5,56). Otro aspecto se vincula con las elaboraciones caseras en los hogares, atravesadas por el dispar gusto por cocinar, contar con habilidades y conocimientos culinarios, recursos materiales (gas, agua, mobiliario, etc.), acceso a diversidad de alimentos frescos y naturales. De ahí que atravesar la cuarentena en los hogares adquiere sentidos diferentes: mientras para sectores más acomodados pudo implicar contar con tiempo para cocinar y comer "mejor", para las entrevistadas de nuestro estudio esta posibilidad se vió limitada.

Todos los testimonios muestran el rol central de las mujeres tanto en la responsabilidad alimentaria al interior de los hogares, como en la gestión de los recursos comunitarios para acceder a alimentos fuera de los hogares. Acordamos con investigaciones que señalan el excesivo énfasis en la responsabilidad individual en los contextos de precarización social y alimentaria, en los que queda vulnerado el derecho a la alimentación ${ }^{(53)}$. Pero la responsabilidad recae con más énfasis en las mujeres, mostrando cómo el modelo patriarcal y la configuración del sistema alimentario actual se sirve de las tareas de cuidado que asumen de manera naturalizada, y que resultan esenciales. Es importante visibilizar el trabajo reproductivo social no remunerado y no reconocido de las mujeres ${ }^{(27,57,58,59)}$. Así, la estrecha relación entre el rol de la mujer y la gestión alimentaria intrahogar muestra que quien asume la carga del hambre son las mujeres.

Emerge la relevancia de las organizaciones de la sociedad civil, actores sociales tradicionalmente claves que fortalecen la disponibilidad y el acceso a los alimentos y comidas en contextos de empobrecimiento ${ }^{(60)}$. Esta red de organizaciones de la sociedad civil, junto a los programas sociales y asistencias alimentarias del ámbito gubernamental, acciones de vecinos autoorganizados contribuyen a mejorar la disponibilidad alimentaria 
en el barrio. Los testimonios manifiestan el papel de relevancia que ocupan estas organizaciones en el contexto de pandemia, aún con escasa infraestructura de cuidado y mínimos recursos económicos, precario nivel de institucionalización o formalización. Los alimentos de los que mayormente disponen para ofrecer, aunque abundantes, son en su mayoría alimentos de elevado aporte de nutrientes críticos, los cuales a su vez contribuyen a reproducir la pobreza y malnutrición, agudizando la vulnerabilidad social y biológica en contexto de pandemia. Esta situación cobra especial importancia en la población de niños, niñas y adolescentes, las principales víctimas ocultas de la pandemia ${ }^{(19,44)}$.

No aparece un cuestionamiento respecto al tipo de alimentos que circulan, aún a sabiendas de la importancia del consumo de alimentos frescos en la dieta cotidiana, tal como lo recomiendan las Guías Alimentarias para la Población Argentina ${ }^{(61)}$, sobre todo en situaciones epidemiológicas donde el buen estado nutricional es clave. Esto conduce a reflexionar sobre la falta de apropiación por parte de sectores de la población de su condición como sujetos de derechos. En las representaciones, "hambre" es no tener alimentos disponibles para ingerir. Sin embargo, no tener más remedio que atiborrarse de harinas y guisos a través de las redes de ayuda y contención pareciera estar naturalizado. Si bien a la pregunta que alude a qué se entiende por una buena alimentación aparecen los frescos a los que precisamente existen mayores dificultades de acceso, existe un silenciamiento sobre este aspecto en sus discursos. Surge la pregunta acerca del lugar del gusto como otra dimensión del derecho a la alimentación, pues cuando se vincula alimentación y pobreza prima la necesidad y no el gusto, recurriendo a la lógica de la optimización para explicar el comportamiento consumidor de los pobres ${ }^{(62)}$. Esto se refleja en el estigma de saberse pobres, en la vergüenza que aparece, en algunos casos, al tener que salir a gestionar recursos alimentarios o admitir, en otros casos, que a veces no queda otra opción que la de alimentarse durante semanas a base de tortas fritas. De ahí las aclaraciones de algunas mujeres que comentan que en sus hogares comen tortas fritas "solo cuando llueve, tal como dicta la tradición". También aparece el estigma de ser inmigrantes de países limítrofes y de origen campesino cuando refieren preparar elaboraciones regionales, y referirse a estas con los apelativos de "pesadas" y "grasosas". Cuando también lo son otras preparaciones, asociadas a clases medias argentinas, como comidas rápidas o semi elaboradas. Esto señala que la desigualdad social y la discriminación de grupos desfavorecidos e identitaria subyacen en la problemática de la malnutrición. Diversos estudios analizan la relación entre inseguridad alimentaria, desigualdad, raza, etnicidad y el género ${ }^{(63)}$, observándose que la "alteridad" es una herramienta de marginación. Las voces de las mujeres paraguayas son deslegitimadas y silenciadas respecto a sus prácticas, habilidades y conocimientos culinarios $^{(64,65)}$. Cabe señalar la importancia por parte de los espacios de toma de decisiones de abordar la etnicidad en las estrategias de superación de la seguridad alimentaria y nutrición. Implica desandar visiones que conciben a las poblaciones en situación de desigualdad como actores pasivos, validar sus sistemas de conocimientos, claves para mejorar la alimentación, fortalecer la diversidad cultural y la construcción de sociedades sin discriminación y exclusión ${ }^{(19)}$.

\section{CONCLUSIONES}

Esta pandemia plantea una emergencia sociosanitaria con impactos directos e indirectos en la malnutrición por exceso, más aún en niños, niñas y adolescentes y grupos vulnerabilizados, en la que se entrecruzan y condicionan la desigualdad, el empobrecimiento, la inequidad de género, las dimensiones de grupos empobrecidos e identidad, y los entornos alimentarios obesogénicos. Los distintos canales de acceso a los alimentos, ya sea la compra directa, la de las redes de asistencia alimentaria oficial y/o de las organizaciones de la sociedad civil, como también 
entre vecinos autogestionados y donaciones alimentarias, en todos los casos predominan alimentos de elevado procesamiento industrial y productos con elevados nutrientes críticos que reproducen la malnutrición. Queda claro el lugar central de las mujeres en la gestión alimentaria dentro y fuera del hogar, que asumen la carga del hambre. A pesar de que el problema de la malnutrición señala su vínculo con el exceso, la preocupación está centralizada en no pasar hambre, y no se discute la calidad de alimentos a los que se accede por los distintos canales. Se detecta una espiral que merece mayor estudio, en la que se conectan la preocupación por el hambre en el contexto de la aparente disponibilidad de alimentos. El problema radica en que se trata de alimentos cuyo consumo se relaciona con el deterioro de la salud y la ausencia de elementos por parte de la población para reclamar, en el marco del derecho, alimentos sanos y adecuados, quedando invisibilizada la problemática de la malnutrición por exceso. En esta espiral se conjugan la responsabilidad individual ante entornos obesogénicos, el empobrecimiento, la condición de sectores empobrecidos y en desventaja, el género y la etnia, percibidos como un estigma del cual se avergüenzan. Al momento del estudio, en el contexto de aislamiento por la pandemia de COVID-19, se evidencia la ausencia de marcos normativos que puedan transformar los ambientes obesogénicos y estructurar sistemas alimentarios que promuevan el acceso a una alimentación saludable. Son claves los cambios sistémicos basados en los derechos humanos para abordar crisis paralelas de alimentos, pandemia y medios de vida, y la necesidad de contar con políticas públicas que promuevan sistemas alimentarios más justos, sostenibles con el ambiente, que democraticen los recursos productivos con canales cortos de distribución. Así se podrá generar mayor igualdad y acceso a alimentos frescos.

\section{FINANCIAMIENTO}

El presente estudio se realizó con financiamiento de UNICEF Argentina. El estudio previo, gracias al cual se generaron los lazos con las entrevistadas y el barrio, realizado entre 2018 y 2019 y titulado "Alimentación y Entornos Obesogénicos: Estudio desde una mirada multidisciplinaria en contextos urbanos y periurbanos de Buenos Aires", recibió el financiamiento del Proyecto UBACyT No 20020170200140BA, Resolución Consejo Superior No 1041/18, Secretaría de Ciencia y Técnica, Universidad de Buenos Aires; y la beca de Investigación Salud Investiga Dr. Abraam Sonis Convocatoria 2018, Dirección de Investigación para la Salud, Secretaria de Salud y Desarrollo Social de la Nación, bajo la coordinación institucional de CISPAN y la participación de FIC y Programa Nutricional de la Ciudad Autónoma de Buenos Aires. Gracias a los lazos entablados a partir de dicho proyecto fue posible volver al barrio en el marco del contexto del COVID-19 en 2020.

\section{CONFLICTO DE INTERESES}

Las autoras declaran no tener vínculos o compromisos que condicionen lo expresado en el texto y que puedan ser entendidos como conflicto de intereses.

\section{AGRADECIMIENTOS}

A Martín Miranda, cuya colaboración en este estudio fue clave; a todas nuestras interlocutoras, en especial, a Ramona, quien tuvo que partir. Todas las personas del barrio $21 / 24$, quienes nos brindaron su tiempo, sentires y experiencias de vida.

\section{REFERENCIAS BIBLIOGRÁFICAS}

1. Naciones Unidas. Informe del Relator Especial sobre el derecho a la alimentación, Olivier De Schutter [Internet]. 2011 [citado 18 jul 2021]. Disponible en:http://tinyurl.com/gomd $44 \mathrm{k}$.

2. Organización Mundial de la Salud. Malnutrición [Internet]. 9 jun 2021 [citado 18 jul 2021]. Disponible en: https://tinyurl.com/3ee9n8jj.

3. Swinburn B , Kraak VI, Dietz WH, et al. The global syndemic of obesity, undernutrition, and climate change: The Lancet Commission report. The Lancet. 2019;393(10173):S791-S846. doi: 10.1016/S01406736(18)32822-8.

4. Organización de las Naciones Unidas para la Alimentación y la Agricultura. Experto internacional identifica a 
Chile como país ejemplo en políticas contra la obesidad [Internet]. 23 ago. 2019 [citado 18 jul 2021]. Disponible en: https://tinyurl.com/3phwhna3.

5. Swinburn B, Egger G, Raza F. Dissecting obesogenic environments: the development and application of a framework for identifying and prioritizing environmental interventions for obesity. Preventive Medicine. 1999;29(6 Pt1):S563-S570. doi: 10.1006/pmed.1999.0585.

6. Vandevijvere S, Chow C, Hall K, Umali E, Swinburn B. Increased food energy supply as a major driver of the obesity epidemic: a global analysis. Bulletin World Health Organization. 2015;93(7):S446-S456. doi: 10.2471/BLT.14.150565.

7. Organización Panamericana de la Salud. Alimentos y bebidas ultraprocesados en América Latina: tendencias, efecto sobre la obesidad e implicaciones para las políticas públicas. Washington DC: OPS; 2015.

8. Crovetto MM, Uauy R, Martins AP, Moubarac JC, Monteiro C. Disponibilidad de productos alimentarios listos para el consumo en los hogares de Chile y su impacto sobre la calidad de la dieta (2006-2007). Revista Médica de Chile. 2014;142(7):850-858.doi: 10.4067/ S0034-98872014000700005.

9. Naciones Unidas. Informe presentado por el Relator Especial sobre el derecho a la alimentación, Olivier De Schutter; Informe final: El potencial transformador del derecho a la alimentación [Internet]. 2014 [citado 18 jul 2021]. Disponible en: http://tinyurl.com/zhzhnoz.

10. Piaggio LR, Solans AM. Enfoques socioculturales de la alimentación: Lecturas para el equipo de salud. Buenos Aires: Akadi; 2014.

11. Merril S, Scott C. Syndemics and public health: Reconceptualizing disease in bio-social context. Medical Anthropology Quarterly. 2003;17(4):S423-S441. doi: 10.1525/maq.2003.17.4.423.

12. Comisión Económica para América Latina y el Caribe. El desafío social en tiempos del COVID-19: Informe Especial COVID-19 No. 3 [Internet]. 12 may 2020 [citado 16 jun 2020]. Disponible en: https://bit.ly/2NfgQKd.

13. Instituto Nacional de Estadística y Censos. Incidencia de la pobreza y la indigencia en 31 aglomerados urbanos: Segundo semestre de 2020. Informes Técnicos [Internet]. 2020;5(59) [citado 16 jun 2020]. Disponible en: https://tinyurl.com/kzjpzc5k.

14. Instituto Nacional de Estadística y Censos. Incidencia de la pobreza y la indigencia en 31 aglomerados urbanos: Segundo semestre de 2019. Informes Técnicos [Internet]. 2020;4(59) [citado 16 jun 2020]. Disponible en: https://tinyurl.com/3yucte22.

15. Instituto Nacional de Estadística y Censos. Canastas básicas en pesos y variación interanual: Gran Buenos Aires, mayo de 2019 y 2020 [Internet]. 17 jun 2020 [citado 16 jun 2020].https://tinyurl.com/29txwbwa

16. Unicef. Unicef actualiza la estimación de pobreza infantil: Alcanzaría a más de 8 millones de chicas y chi- cos [Internet]. 5 ago 2020 [citado 18 jul 2021]. Disponible en:https://tinyurl.com/ys62xxxa.

17. Argentina, Ministerio de Salud. $2^{\circ}$ Encuesta Nacional de Nutrición y Salud: Resumen ejecutivo [Internet]. Buenos Aires: Ministerio de Salud; 2019 [citado 18 jul 2021]. Buenos Aires, Argentina. Disponible en: https:// tinyurl.com/2cxw3fvh.

18. Unicef. La pobreza y la desigualdad de niñas, niños y adolescentes en la Argentina. Efectos del Covid-19 [Internet]. Buenos Aires: UNICEF Argentina; 2020[citado 16 jul 2020]. Disponible en: https://tinyurl.com/2p8e7s85.

19. FAO, FIDA, OPS, WFP, Unicef. Panorama de la seguridad alimentaria y nutricional en América Latina y el Caribe 2020 [Internet]. Santiago: FAO, OPS, WFP, UNICEF; 2020[citado 16 jul 2020]. Disponible en:https://doi. org/10.4060/cb2242es

20. United Nations System Standing Committee on Nutrition. Food Environments in the COVID-19 Pandemic [Internet]. 2020 [citado 10 jul 2020]. Disponible en: https://tinyurl.com/npmmvxvf.

21. Fischler C. El (h)omnívoro: el gusto, la cocina, el cuerpo. Barcelona: Anagrama; 1995.

22. Contreras J, Gracia-Arnaiz M. Alimentación y cultura: Perspectivas antropológicas. Barcelona: Ariel; 2005.

23. Mintz S. Sabor a comida, sabor a libertad. México DF: CIESAS; 2003.

24. FAO, OMS, OPS.Panorama de la Seguridad Alimentaria y Nutricional en América Latina y el Caribe 2016: Sistemas alimentarios sostenibles para poner fin al hambre y la malnutrición. Santiago: FAO, OMS, OPS; 2017.

25. FAO, OPS, WFP, Unicef. Panorama de la seguridad alimentaria y nutricional en América Latina y el Caribe 2019 [Internet]. 2019 [citado 16 jul 2020]. Disponible en:https://tinyurl.com/mr3zwuct.

26. D'Argemir DC. Los cuidados y sus máscaras: Retos para la antropología feminista. Mora. 2014;20:167-182.

27. Carrasco C, Borderías C, Torns T. Introducción: El trabajo de cuidados, Antecedentes históricos y debates actuales. En: El trabajo de cuidados: Historia, teoría y política. Madrid: Catarata; 2011. p. 13-97.

28. Crenshaw K. Dermarginalizing the intersection of race and sex: A black feminist critique of antidiscrimination doctrine, feminist theory, and antiracist politics. Chicago: University of Chicago Legal Forum; 1989. p. 139-197.

29. Kessler G. Las consecuencias de la estigmatización territorial: Reflexiones a partir de un caso particular. Espacios en Blanco. 2012;(22):165-197.

30. Goffman E, Guinsberg L. Estigma: la identidad deteriorada. Buenos Aires: Amorrortu, 1970.

31. Bayón M. La construcción del otro y el discurso de la pobreza: Narrativas y experiencias desde la periferia de 
la ciudad de México. Revista Mexicana de Ciencias Políticas y Sociales. 2015;60(223):357-376.

32. Walsh C. Interculturalidad crítica y educación intercultural. Construyendo Interculturalidad Crítica. 75;96(2010):167-181

33. Dirección General de Estadística y Censos. Resultados provisionales del Censo Nacional de Población, Hogares y Viviendas 2010 en la Ciudad de Buenos Aires [Internet]. [citado 25 jul 2020]. 2011 [citado 16 ju 2020]. Disponible en: https://tinyurl.com/yd7pnw7v.

34. Autoridad de Cuenca Matanza Riachuelo. Informe final EISAAR Camino de Sirga del Barrio Villa 21-24, CABA [Internet]. 2018 [citado 25 jul 2020]. Disponible en: https://tinyurl.com/2p8aeuhn.

35. Molek N. Procesos identitarios entre los migrantes eslovenos de entreguerras y sus descendientes en Argentina. La Rivada. 2016;4(7):3-21.

36. Guber R. La etnografía: método, campo y reflexividad. Bogotá: Grupo Editorial Norma; 2001.

37. Asociación Médica Mundial. Declaración de Helsinki. Declaración de Helsinki de la AMM - principios éticos para las investigaciones médicas en seres humanos [Internet]. 2017 [citado 25 jul 2020]. Disponible en: https://tinyurl.com/2p896vsm.

38. Guber R. El salvaje metropolitano: reconstrucción del conocimiento social en el trabajo de campo. Buenos Aires: Paidós; 2004

39. Administración Nacional de Seguridad Social. Asignación Universal por Hijo [Internet]. 2020 [citado 25 ju 2020]. Disponible en: https://tinyurl.com/pj7bw6k8.

40. Administración Nacional de Seguridad Social. Ingreso Familiar de Emergencia [Internet]. 2020 [citado 25 jul 2020]. Disponible en: https://tinyurl.com/3t5wj5ps.

41. Administración Nacional de Seguridad Social. Tarjeta Alimentar [Internet]. 2020 [citado 25 jul 2020]. Disponible en: https://tinyurl.com/2p9acp4s.

42. Gobierno de la Ciudad de Buenos Aires. Canasta Escolar Nutritiva (Covid-19) [Internet]. 2020 [citado 22 jul 2020]. Disponible en: https://tinyurl.com/mry5mvzv.

43. Aguirre P.Patrón alimentario, estrategias de consumo e identidad en la Argentina, 1995. En: Pinotti LV, Álvarez M. Procesos socioculturales y alimentación. Buenos Aires: Edición del Sol; 1997. p. 161-187.

44. Universidad Católica Argentina, Observatorio de la Deuda Social Argentina. Efectos del ASPO-COVID-19 en el desarrollo humano de las infancias argentinas. Informe de avance: Diciembre 2020 [Internet]. 2020 [citado 16 jul 2020]. Disponible en: https://tinyurl.com/yszacax8.

45. Fundación Heinrich Böll, Fundación Rosa Luxemburg, Grupo de Ecología del Paisaje y Medio Ambiente. El atlas del agronegocio [Internet]. 2018 [citado 15 may 2021]. Disponible en: https://tinyurl.com/4rwydbpw.
46. Argentina. Ley 27642 Promoción de la Alimentación Saludable [Internet]. 2021 [citado 30 dic 2021]. Disponible en: https://tinyurl.com/yetcaavf.

47. Piaggio LR. El derecho a la alimentación en entornos obesogénicos: Reflexiones sobre el rol de los profesionales de la salud. Salud Colectiva. 2016;12(4):605-619. doi: 10.18294/sc.2016.934.

48. Aguirre P. Comida, cocina y consecuencias: la alimentación en Buenos Aires. En: Torrado S. Población y bienestar en la Argentina del primero al segundo centenario: Una historia social del siglo XX. Buenos Aires: Edhasa; 2007.

49. Swinburn B, Shelly A. Effects of TV time and other sedentary pursuits. International Journal of Obesity. 2008;32(Suppl 7):S132-S136. doi: 10.1038/ijo.2008.249.

50. Monteiro C, Bertazzi Levy R, Claro Moreira R, De Castro I, Cannon G. A new classification of foods based on the extent and purpose of their processing. Cadernos de Saúde Pública. 2010;26(11):2039-2049. doi: 10.1590/S0102-311X2010001100005.

51. Organización Mundial de la Salud. Conjunto de recomendaciones sobre la promoción de alimentos y bebidas no alcohólicas dirigida a los niños. Ginebra: OMS; 2010.

52. Balan D, Palacios A, Bardach A, Rodriguez B, Ciapponi A, Garcia Marti S, Perelli L, Augustovski F, Gittens-Baynes KA, Vianna C, Guevara G, Pichon-Riviere A, Alcaraz A. Políticas fiscales para reducir el consumo de bebidas azucaradas: Evidencia para la implementación de políticas públicas en Latinoamérica y el Caribe (Documento Técnico Nº 54). Buenos Aires: Instituto de Efectividad Clínica y Sanitaria; 2019.

53. Arnaiz MG, Demonte F, Bom Kraemer F. Prevenir la obesidad en contextos de precarización: respuestas locales a estrategias globales. Salud Colectiva. 2020;17:e2838. doi: 10.18294/sc.2020.2838.

54. Zapata ME, Rovirosa A, Carmuega E. Cambios en el patrón de consumo de alimentos y bebidas en Argentina, 1996-2013. Salud Colectiva. 2016;12(4):473-486.

55. McMichael P. A food regime analysis of the 'world food crisis'. Agriculture and Human Values. 2009;26:281. doi: 10.1007/s10460-009-9218-5.

56. ETC Group. Who Will Feed Us? [Internet]. 15 oct 2017 [citado 15 may 2021]. Disponible en: https://tinyurl.com/2wwcrmjf.

57. Esquivel V, Faur E, Jelin E. Las lógicas del cuidado infantil: Entre las familias, el Estado y el mercado. Buenos Aires: IDES; 2012

58. Esteban ML. Crítica del pensamiento amoroso. Barcelona: Bellaterra; 2011.

59. Ali S, Mirza H, Phoenix A, Ringrose J. Intersectionality, Black British feminism and resistance in education: a roundtable discussion. Gender and Education. 2010;22(6):647-661. 
60. Fernández GJ. Experiencias de cuidados comunitarios en tiempos de Pandemia. Margen. 2020;(98):1-7.

61. Argentina, Ministerio de Salud, Dirección de Promoción de La Salud y Control de Enfermedades No Transmisibles. Manual para la aplicación de las guías alimentarias para la población argentina [Internet]. 2018 [citado 16 jul 2020]. Disponible en: https://tinyurl. com/2p8s9d6w.

62. Cattáneo A. Alimentación, salud y pobreza: la intervención desde un programa contra la desnutrición. Archivos Argentinos de Pediatría. 2002;100(3):222-232.
63. Woods D. Mujeres invisibles: hambre, pobreza, racismo y cuestiones de género en el Reino Unido [Internet]. 2019 [citado 16 jul 2020]. Disponible en: https:// tinyurl.com/2v8fnd57.

64. Mueller JC. Producing colorblindness: Everyday mechanisms of white ignorance. Social Problems. 2017;64(2):219-238. doi: 10.1093/socpro/spw061.

65. Mirza H. Black British feminism then and now. Media Diversified [Internet]. 13 mar 2014 [citado 16 jul 2020]. Disponible en: https://tinyurl.com/3asuu8xy.

\section{FORMA DE CITAR}

Sammartino G, Naumann SA. "Nosotros somos de harina": Prácticas y significados alimentarios en la Villa 21-24 de la Ciudad Autónoma de Buenos Aires, en contextos de COVID-19. Salud Colectiva. 2022;18:e3730. doi: 10.18294/ sc. 2022.3730 .

Recibido: 29 sep 2021 | Versión final: 31 dic 2021 | Aprobado: 15 feb 2022 | Publicado en línea: 2 mar 2022 